\title{
Corela
}

Cognition, représentation, langage

HS-33 | 2020

Textuel, textiel. Repenser la textualité numérique

\section{Aux origines du textiel. Entretien avec Marie Després-Lonnet}

Ingrid Mayeur et Marie Desprès-Lonnet

\section{(2) OpenEdition}

Journals

Édition électronique

URL : https://journals.openedition.org/corela/11756

DOI : 10.4000/corela.11756

ISSN : $1638-573 \mathrm{X}$

Éditeur

Université de Poitiers

Référence électronique

Ingrid Mayeur et Marie Desprès-Lonnet, «Aux origines du textiel. Entretien avec Marie Després-Lonnet », Corela [En ligne], HS-33 | 2020, mis en ligne le 26 novembre 2020, consulté le 07 décembre 2022.

URL : http://journals.openedition.org/corela/11756; DOI : https://doi.org/10.4000/corela.11756

Ce document a été généré automatiquement le 14 juillet 2021.

\section{(c) (i) (2) (2)}

Creative Commons - Attribution - Pas d'Utilisation Commerciale - Partage dans les Mêmes Conditions 4.0 International - CC BY-NC-SA 4.0

https://creativecommons.org/licenses/by-nc-sa/4.0/ 


\title{
Aux origines du textiel. Entretien avec Marie Després-Lonnet
}

\author{
Ingrid Mayeur et Marie Desprès-Lonnet
}

IM. À quelle occasion le concept de textiel a-t-il été forgé ? Dans quel contexte de recherche, et pour répondre à quels besoins?

MDL. C'est au démarrage d'un projet intitulé "Métamorphoses médiatiques ", financé et soutenu par le CNRS et le centre d'études et de recherches de la BPI que nous avons tracé collectivement les grandes lignes de ce tournant méthodologique les conclusions des études que nous avons menées dans ce cadre ont fait l'objet d'une publication collective, Lire, écrire, récrire: Objets, signes et pratiques dans les médias informatisés (Davallon et al. [2003a] 2013). Notre projet était alors centré autour des usages des «médias informatisés" et des "écrits de réseau ». Nous voulions les aborder et les interroger en tentant de sortir des fausses évidences méthodologiques qui reposent souvent sur des présupposés qui empêchent de «voir » ce qui se passe vraiment. Nous cherchions plus particulièrement à rompre avec une analyse des processus de communication basée sur le modèle fonctionnaliste «émission/ réception ». Notre objectif scientifique était d'expérimenter des approches qui nous permettraient de qualifier la circulation, la transformation et donc les métamorphoses des textes et des savoirs, autrement qu'en les pensant à partir de rapports (de force, de pouvoir, de résistance...) entre des sphères qu'il serait possible d'opposer ou de considérer indépendamment tout en mesurant des effets. Il s'agissait de mener une réflexion théorique, tout en imaginant de nouvelles approches méthodologiques pour nos enquêtes de terrain. Pour changer de regard sur les phénomènes que nous voulions observer, nous devions les construire différemment en les envisageant plus globalement, dans leurs dimensions technique, sociale et symbolique ${ }^{1}$.

Nous étions confrontés à l'époque à une aporie terminologique lorsqu'il fallait nommer les choses que nous analysions. Pouvait-on appeler ce qui s'affichait à l'écran une " page »? Comment qualifier les activités menées par les personnes que nous observions? De quoi le concept d'hypertexte rendait-il compte du point de vue de la lecture et des processus d'interprétation? Jean-Louis Weissberg proposait par 
exemple de nommer lect-acteur celui qui tout à la fois lisait et devait «activer » le texte pour qu'il apparaisse à l'écran. J'ai donc proposé le terme textiel qui est la contraction de texte et de logiciel pour désigner ces textes/outils mais également ces textes/codes. Le logiciel est programmé pour permettre à celui qui l'utilise d'agir sur l'ordinateur et le textiel désigne ce qui tout à la fois se lit et s'utilise ou peut être, pourrait-on dire, se laisse agir. Le concept de textiel est une tentative de rendre compte de la dimension à la fois textuelle et opératoire des écrits concernés. Il y a une forme de réciprocité : la lecture sollicite l'action et l'action permet la lecture (par l'activation, l'affichage d'autres textes).

Il n'y a pas que le concept de textiel qui a été imaginé à ce moment-là ; Emmanuël Souchier a par exemple proposé le concept de "signe passeur " pour désigner les signes sur lesquels on peut cliquer (qui selon la définition qu'il en donne "postule l'inter-détermination des modalités d'écriture, du support et des outils, des dispositifs techniques et des pratiques sociales d'écriture "). Nous nous sommes intéressés, au cours d'un autre projet collectif dirigé par Jean Davallon, aux «traces d'usage » (Davallon 2012), terme qui désigne le projet de certains textes à intégrer ou solliciter d'autres actions ou échanges. Nous avons également réfléchi aux métamorphoses médiatiques et à ce qu'Yves Jeanneret a proposé d'appeler alors les "médias informatisés» (Tardy et Jeanneret 2007). Nous avons étudié les nouvelles pratiques d'écriture dans différents contextes sociaux et professionnels. J'ai travaillé avec Dominique Cotte sur le processus de production du journal télévisé : de la conférence de rédaction au passage à l'antenne, ce qui nous a permis de montrer en quoi les nouveaux outils "textualisaient " les pratiques en obligeant à nommer, désigner, décrire des objets, des moments, des acteurs, des activités qui jusque-là ne le nécessitaient pas. Nous avons aussi suivi les différentes étapes et les différents scripteurs (y compris informatiques) qui participaient à la fabrication d'une émission pour mettre en lumière la multiplicité et la variété des discours qui s'entremêlent et qui circulent dans des états intermédiaires, entre les différents acteurs internes et externes, impliqués dans le processus, mais également le rôle prescripteur des environnements techniques qui obligent à certains formatages, certaines organisations textuelles (Després-Lonnet et Cotte 2007). Je me suis ensuite intéressée aux processus interprétatifs et à ce qui « fait » document, ce qui m'a permis de mettre en lumière les problèmes que pose la délocalisation des contextes de production, lorsque les textes circulent via les réseaux. J'ai notamment observé des enfants de classes primaires confrontés à des résultats de recherche obtenus dans des moteurs tels que Google et qui peinaient à comprendre « qui » leur parlait.

C'était un moment d'ébullition, nous étions une vingtaine, au sein d'un groupe à géométrie variable. Nous avons travaillé ensemble au moins une dizaine d'années sur cette question des médias informatisés, autour d'une sorte de noyau dur, avec, à chaque fois, des chercheurs qui nous ont rejoints et qui nous ont quittés. Il y avait là l'idée de réaliser un travail collectif autour de cette question, avec des approches et des regards complémentaires, mais de le réaliser conjointement. L'autre idée était d'avoir une approche interdisciplinaire : Yves Jeanneret et Emmanuël Souchier sont au départ des littéraires, il y avait des collègues qui se situaient du côté de la médiation culturelle, Joëlle Le Marec plutôt en ethnologie, et moi j'étais plutôt en médiation des savoirs, avec un intérêt très vif pour le substrat technique. L'idée était de s'inscrire dans une interdiscipline, en étant chacun spécialiste, et en ayant une 
interconnaissance qui nous permettait, dans les séminaires ou dans le travail de terrain, non pas d'être spécialiste de tout, mais de nous appuyer collectivement sur les compétences de chacun.

Vous avez donc une double formation, en informatique et en sciences de l'information et de la communication?

En effet. Cela me plaçait dans une position assez intéressante pour mes collègues : je pouvais guider la compréhension du substrat technique, expliciter les couches techniques derrière le média informatisé, ce qui leur permettait de s'y intéresser ou non. Mais de pouvoir s'y intéresser s'ils le souhaitaient; par exemple, quand nous avons forgé le concept d'architexte, cela a donné lieu à des débats : de quel point de vue est-ce qu'on parle d'architexte, est-ce que c'est l'architexte tel que l'usage le désignerait, soit: la personne qui se trouve face à l'écran voit-elle une différence entre le texte d'une part, et ce qui lui donnerait accès à la fois techniquement et conceptuellement au texte d'autre part; ou est-ce qu'on peut aussi comprendre les sous-couches techniques, comme le langage $h t m l$, ou les bases de données, et tenter de voir en quoi cela explique le textiel ? Cela permet d'appréhender « l'autre côté du miroir ", comment se tissent les liens entre ce qui, finalement, s'affiche comme un texte à l'écran, et tout ce qu'il y a derrière, les modalités techniques de fabrication, de préfabrication.

Comment l'environnement numérique agit-il sur les frontières du texte, envisagé comme textiel ? Sont-elles constamment mouvantes ou tolèrent-elles des formes de stabilisation (permanentes, momentanées, etc.) et, dans ce cas, avec quels éléments de structuration (écran, page web, site, etc.)?

Je distinguerais assez volontiers ce qui se passe techniquement et ce que les choses qui s'affichent à l'écran signifient pour nous. Si par exemple j'utilise un traitement de textes pour écrire un poème et que j'imprime ce poème, d'un point de vue matériel, je dispose d'une forme (stabilisée par l'inscription matérielle) de ce texte. Mais dans le même temps, le fichier qui contient le code qui permet à ce texte de s'afficher à l'écran existe toujours et peut être en permanence modifié. Cette modification peut, elle aussi, être envisagée selon différentes modalités : si j'ouvre le fichier dans le traitement de textes qui a servi à le produire, le texte du poème, ce dernier va s'afficher à l'écran sous une forme visuelle relativement proche de celle qu'il a sur le papier. Mais je pourrai aussi intervenir directement dans le code du fichier grâce à un autre logiciel qui me donnerait accès au code. Ce même texte ne sera alors pratiquement pas lisible pour lui-même, même si, dans l'entrelacs de ce qui s'affiche à l'écran (et qui est, en définitive, aussi un texte) je retrouverai ici et là des bribes de mon poème que je pourrai modifier selon d'autres modalités (certes beaucoup plus compliquées mais possibles quand même...). Je travaille beaucoup avec des informaticiens ou des mathématiciens qui utilisent des plateformes de développement de sites web ou encore le langage LaTeX pour rédiger leurs articles ou divers documents et il est clair qu'ils écrivent avec une représentation duale des textes qu'ils produisent : le texte est à la fois « dans » ce qu'ils codent (avec une mise en abîme puisqu'ils sont en train de produire un « autre » texte dans le langage codé qu'ils utilisent) et (par une forme de représentation mentale anticipatoire) la forme que ce texte va prendre lorsqu'il s'actualisera dans d'autres environnements techniques et matériels. Les mathématiciens trouvent par exemple plus simple de programmer les figures que de les dessiner ou de coder les formules et les fonctions mathématiques que de devoir les mettre en forme eux-mêmes. 
Un site web est-il un texte? Un livre est-il un texte ? La une d'un journal papier estelle un hypertexte ? Le site Wikipédia est-il une encyclopédie ? C'est un peu la même problématique pour moi et je penche de plus en plus pour une approche pragmatique et constructiviste de la question. Qu'est-ce que c'est pour celui que j'observe? Comment peut-on appréhender les frontières d'un site? Encore une fois, si je suis informaticien et que j'ai développé ce site, je peux dire où il est dans la machine, dans quels répertoires, sur quels serveurs se trouvent les multiples fichiers qui le constituent matériellement, mais dès que je m'intéresse à ce qui se passe à l'écran, le concept même de site devient autre chose. Si je visite le site d'un journal télévisé, la charte graphique du site doit normalement me permettre de situer les textes que j'y lis et d'en comprendre l'organisation, dans ce contexte éditorial. Il y a une métastabilisation architextuelle; comme c'est le cas lorsque j'achète un journal. Il m'est en revanche impossible de cerner précisément la globalité, la profondeur du site. Je dispose d'outils : ce fameux textiel m'offre des potentialités d'activités (écrire, passer à une autre page, démarrer la lecture d'une vidéo, cliquer sur un "bouton ", etc.) et ce sont mes compétences interprétatives, ma capacité à savoir « où » je suis, qui me permettent de situer ces écrits dans des contextes sociaux qui, de mon point de vue, sont les formes de stabilisation/structuration dont nous avons besoin pour faire quelque chose avec ces textes, ne serait-ce que pour les lire.

Ce qui est fondamental pour nous en SIC, c'est le moment où se construit le sens, soit quand il y a la rencontre entre ce projet de lecture et l'actualisation du texte dans une matérialité qui est propre à cet usage-là. Par exemple, si vous manipulez un Kindle, vous manipulez un ebook, un livre électronique : c'est une certaine matérialité qui est très mimétique de la matérialité livresque. Que vous lisiez ce même texte sur votre téléphone, ou sur un écran d'ordinateur, cela active finalement, du point de vue de ce que ça représente pour vous, la même matérialité. D'où la question : face à quoi les outils d'appréhension matérielle et d'action sur les outils de mise à disposition de ce texte, vous font-ils vous sentir? Est-ce que cela mime vraiment une lecture livresque, parce vous avez une pseudo-page cornée sur la droite, et que vous pouvez feuilleter l'ebook en actionnant ce signe indiciel d'une possible post-page ? L'idée est que cela métaphorise le livre...

Cela reprend des imaginaires, qui sont liés à des savoirs procéduraux, des savoir faire acquis dans d'autres domaines de la culture écrite?

Exactement! Des savoirs procéduraux, qui sont référés à des objets matériels autres que l'écran, et qui posent toujours la question de savoir quelle métaphore est sollicitée dans ce qu'on appelle aujourd'hui de façon très agaçante pour moi les UX, c'est-à-dire l'expérience utilisateur ; soit, en face de quel type de pratique est-on en train de vous mettre : pratiques de lecture du livre, scrolling, ou est-ce qu'on est en train de fabriquer un livre avec un logiciel de $\mathrm{PAO}^{2}$ par exemple, tout ça, c'est finalement différentes matérialités, qui sont des matérialités de l'usage du texte. Cela implique aussi la forme sous laquelle on vous sollicite, en tant que lecteur/acteur, vis-à-vis de ce texte.

En effet, dans le collectif Lire, écrire, récrire (Davallon et al. [2003a] 2013), auquel vous avez collaboré, le concept de textiel apparaît pour mettre en évidence le fonctionnement opératoire des écrits numériques qui, en tant qu'ils associent une part langagière et une part technique, sollicitent l'implication active du lecteur dans la composition d'un texte pouvant lui servir (Davallon, Noël-Cadet, et Brochu [2003] 2013, § 96). Le texte comme unité informationnelle prendrait ainsi sens au regard d'un usager et de ses besoins. Si l'on vous 
suit bien, le « faire texte » se joue à deux niveaux : il y a d'une part celui de l'architexte, l'outil technique qui va produire le texte, et d'autre part celui du lecteur et de ses actions qui va le constituer?

Oui, de son projet aussi, son intention, ce vers quoi il va. Quel est son projet d'usage ? Il y a une intention dans la mise à disposition du texte, ce texte est intentionnellement mis à disposition selon une certaine modalité. Cette modalité rencontre un projet d'usage qui fait que l'actualisation va s'effectuer sur le registre de l'intercompréhension entre la proposition et son actualisation dans l'usage réel. Ce que cela change par rapport à une matérialité " papier », c'est que cette dernière permet de figer des moments, de signifier à un moment donné la clôture de l'œuvre : ici, le livre est fini, et il ne va plus s'étendre, sauf si vous l'annotez en tant que lecteur. Mais du point de vue de la proposition qui vous est faite, il y a un moment où cette proposition est figée dans un objet. Tandis que dans le numérique, cette proposition s'actualise en un certain sens (dans un Kindle, il y a un fichier), mais reste en même temps un ensemble d'éléments de textualité potentiels, qui pourraient à n'importe quel moment devenir autre chose, faire une autre proposition. Et c'est à cela, pour moi, qu'il faut être attentif : il y a des formes de stabilisation parce que, en tant qu'être humain, on doit donner un sens à ce qu'on fait et, par conséquent, vous êtes face à des objets que vous devez considérer comme étant stables, parce que vous devez faire quelque chose avec. Cela me fait penser à Roger Rabbit, avec l'épée ; vous vous emparez de cette épée, elle a quand même intérêt à rester une épée quand vous vous battez avec et là, vous vous retrouvez avec une épée chantante, c'est embêtant. Ici, c'est la même chose : quand j'ai le projet de lire un livre, et qu'on me présente à l'écran quelque chose qui a tout l'air d'être ce que je suis en train de vouloir, j'ai quand même un moment de stabilité, je suis en train de lire ce livre. Et donc il y a des formes de stabilisation, qui sont des moments où ce qui apparaît à l'écran correspond à une activité que je vais embrayer par rapport à cette proposition : tout n'est pas constamment mouvant. Ces potentialités s'actualisent, parce que sinon elles n'existent pas : ce sont des potentialités électroniques, des fichiers : il y a bien un moment où il faut que quelque chose devienne perceptible à nos sens, et que cela fasse sens en même temps : à ce moment-là, cela devient un texte. Et c'est un texte parce que c'est nous qui, socialement, le définissons comme texte.

Pourriez-vous préciser en quoi l'architexte, au sens où vous l'entendez, participe du processus de production des textes? Est-ce qu'il crée des bornes formelles, une mise en cohérence des unités compositionnelles du texte, etc. ? En d'autres termes, quelles sont les relations entre architexte et textiel, au regard de la production du texte et du faire texte?

L'idée de l'architexte, c'est de distinguer ce qui fait texte ; sur l'écran, au niveau de ce qui est affiché, quelle est la part de ce qui serait le texte, quelle est la part de ce qui permet d'appréhender le texte ${ }^{3}$. Que ce soit pour le lire, l'écrire, l'activer, le modifier, pour faire quelque chose avec, vous avez autour de ce texte un ensemble sémiotique, un système de signes, qui est l'architexte: à moment donné, il crée à la fois la frontière entre ce qui fait texte et ce qui fait outillage autour du texte, et en même temps, il est ce qui situe, une fois encore, ce texte dans un certain projet. C'est-à-dire que vous avez un texte qui s'affiche sur un traitement de texte; l'architexte, c'est tout ce qui est autour de ce que vous êtes en train de produire comme texte. Si l'on prend une page blanche, au lieu d'avoir une machine à écrire autour de la page blanche, on a l'architexte, qui est l'ensemble des choses qui en creux désignent le 
texte - parce que le texte, c'est ce qui n'est pas autour - et, en en même temps, vous dit ce que vous êtes en train d'être par rapport au texte.

Et cela, aussi bien en pôle production qu'en pôle réception?

Absolument. C'est même important, puisqu'on est dans les textiels, donc des textes activables, qui engagent dans des processus qui sont toujours des processus de lecture/écriture. Là, vous avez la possibilité d'intervenir, vous aussi, dans le texte. Les premiers projets d'hypertexte, dans le sillage du projet de Vannevar Bush ${ }^{4}$, n'étaient pas du tout ce qu'on a aujourd'hui. Les premiers outils d'hypertexte étaient des outils où nous produisions nous-même, facilement, sans avoir à entrer dans le code, de l'hypertexte ; il s'agissait plutôt de la mise en lien de textes, plutôt que ce qu'on a aujourd'hui, soit des liens qui nous sont pré-proposés. C'est davantage, à l'origine, un outil conceptuel pour le créateur du texte qu'un outil de lecture et de passage - ce que Souchier appelle les signes passeurs : et moi je ne suis pas non plus d'accord avec passeurs, mais c'est un débat que j'ai avec lui, on en rit tout le temps.

La présente livraison entend interroger le «faire texte » au sein des écrits numériques dans une perspective linguistique, inspirée des travaux en linguistique textuelle de J.-M. Adam (Adam 2011, 2015). En SIC, la notion de textiel semble surtout s'appliquer au document en tant qu'unité de circulation du savoir; le langage apparaît comme l'une des couches technosémiotiques du texte possédant, dans l'environnement numérique, une épaisseur technique (Davallon, Noël-Cadet, et Brochu [2003] 2013, § 115). Les SIC ont-elles à un moment donné considéré la matérialité langagière comme contribuant au processus de production du texte?

J'ai peur de ne pas comprendre votre question. Les SIC s'intéressent à la communication et donc aux conditions sociales de production, de circulation et d'interprétation de discours. Pour nous les discours sont d'abord des formes langagières et textuelles, dont la matérialité dit quelque chose des intentions de ceux qui les ont fabriqués mais aussi de ce que cette matérialité qui lui est propre dit de l'autre auquel ce texte est destiné. Si on relit Umberto Eco, on sait bien que dans le geste d'écriture, il y a quelque chose qui est de l'ordre du Lecteur Modèle, mais en même temps, le Lecteur Modèle, c'est à la fois les implicites dans tout ce qui est dit, mais également dans la manière dont c'est dit. Et cela peut aller probablement jusqu'à la manière dont c'est mis en forme.

Pour le dire autrement, nous n'avons pas vraiment eu le sentiment que les SIC s'étaient intéressées aux questions de liage textuel, de reprise anaphorique, de formation de la cohérence textuelle en pôle écriture dans le texte numérique, etc. Est-ce néanmoins le cas, ou cette discipline considère-t-elle la matérialité langagière comme englobée dans un ensemble beaucoup plus large, ainsi que vous semblez le concevoir?

Je pense que c'est plutôt cela. Par exemple, nous avons travaillé sur la question de la vulgarisation scientifique, et ce qu'on constate, c'est une hybridation médiatique : par exemple, si vous allez sur le site de la Cité des Sciences, du Palais de la découverte ou d'un Muséum quelconque, vous trouverez à la fois des vidéos, des textes, etc. Je pense que l'entrée par le texte risque de ne pas tenir compte de cette intermédialité. La complexité de ce qui s'affiche à l'écran va bien au-delà. Quand nous avons travaillé sur les traces d'usage par exemple, Jean Davallon, entre autres, s'est intéressé aux sites médiateurs du site Gallica (Davallon, Noël-Cadet, et Brochu [2003] 2013); soit des sites qui citaient, d'une certaine manière, avec des liens hypertexte, le site de Gallica. Les chercheurs se sont intéressés (même si c'est plutôt de la sémiologie) à la manière dont était énoncée la possibilité d'aller sur le site Gallica, en concevant cela comme 
une proposition d'usage, une trace d'usage, soit : qu'est-ce que les gens qui avaient mis ce texte à disposition avaient comme rapport avec le site Gallica ? Et cela passait tout de même par l'analyse du discours. Donc on ne fait pas que cela; on fait de l'analyse de discours, mais pas uniquement. Si on ne fait que de l'analyse de discours, cela permet d'attraper quelque chose mais ça ne permet pas d'attraper tout; si je me limite aux formulations, à la manière dont le texte est fabriqué, je vais manquer une grande part de ce que je regarde, et nous, ce que l'on regarde, ce sont les modalités de la communication. Le texte en est une partie, mais ce n'est pas la seule. En sciences de l'information et de la communication, on peut faire de l'analyse du discours mais cette analyse du discours, elle va être mise au service d'une question de médiation ou de communication.

La notion de textiel semble s'être éteinte depuis ces premières recherches menées dans le courant des années 2000, étant donné qu'elle ne paraît pas couramment utilisée dans les travaux actuels en SIC et est ignorée en sciences du Langage. Y a-t-il une raison précise à cela?

C'est un concept qui nous a semblé particulièrement heuristique à l'époque et je pense que nous avons ensuite voulu préciser les choses, tant du point de vue des matérialités des objets numériques que des usages. Pour nous, cela a été extrêmement intéressant et important au moment où on l'a forgé, pour tenir compte de cette spécificité, de ce double statut de texte et de logiciel : cela permettait de tenir compte de ces propositions qui allaient au-delà de la lecture, soit une proposition de lecture-usage; une lecture, et en même temps une lecture active, c'est-à-dire une lecture activant aussi un certain nombre de dispositifs qui étaient, à la fois, intégrés dans l'activité de lecture, mais pas uniquement.

En quelque sorte, vous étiez partis d'un concept globalisant qui vous avait permis de rendre compte de quelque chose à un moment donné, et vous avez ensuite affiné des composantes, des formes d'unités compositionnelles de ce qui «fait » textiel (signes passeurs, architexte, etc.), de tout ce qui permet l'opérativité du textiel ?

Voilà, c'est tout à fait ça. L'idée du textiel continuerait d'être intéressante pour signifier que tout texte numérique aujourd'hui porte en lui cette potentialité d'activation de quelque chose, quel que soit le quelque chose en question. Ensuite, effectivement, il devient nécessaire de passer au-delà de cela : d'accord, il y a cette spécificité, mais après, cela intègre tout de même, encore une fois, des univers de sens, des pratiques sociales, des environnements qui font que ce textiel va devenir quelque chose, et qu'il est intéressant de comprendre le sens que cela va avoir pour ceux qui s'en emparent. Le texte numérique a déjà comme pré-qualité d'être un textiel ; ensuite, on va tenter de comprendre au-delà, ce qu'il devient pour celui qui s'en empare, du point de vue de son contenu, et du point de vue de ce qu'il propose de faire avec. Après, ce à quoi on s'est intéressés, au-delà de l'architexte, ce sont aussi toutes les questions d'organisation des savoirs qui sont en amont, un peu comme si on était passés des pages du livre à la bibliothèque. À un moment donné, ces textes apparaissent à l'écran, mais il y a des modalités qui sont préalables et nécessaires : on tape dans Google, on fait des recherches préparatoires : il y a donc des métadonnées, des discours qui sont tenus sur ces textes, peu lisibles à l'écran, et qui sont finalement ce par quoi on les attrape, ce par quoi ils deviennent accessibles en ligne. De ce fait, je me suis davantage intéressée à cette question-là par la suite, ainsi qu'au projet de web sémantique, et à ce qu'on appelle le Big Data. Dans cet ordre d'idées, nous nous sommes occupés de cette question de la textualisation, soit, au-delà du texte (enfin, 
dans son ancienne acception), tout ce qui va être produit comme matière textuelle, qui n'était pas nécessaire quand les textes étaient dans des matérialités papier, et qu'il devient nécessaire d'ajouter: toutes ces métadonnées, tous ces textes sur le texte, qu'on a appelés la textualisation des pratiques. On a désormais la nécessité de nommer des choses qu'avant on n'avait pas besoin de nommer, pour arriver à faire les choses qu'on voudrait faire avec.

Et vous vous êtes intéressés à la manière dont ces pratiques sociales textualisées se liaient aux textes auxquels elles s'associaient?

Oui, cela veut dire que l'on crée, en même temps que le texte, un méta-texte : ce n'est pas l'architexte mais ce sont les métadonnées, ce à partir de quoi on peut revenir au texte. À partir du moment où vous avez comme projet d'enregistrer un texte, dans la machine, alors vous devez produire des métadonnées. Avant, vous mettiez votre papier dans la machine à écrire, ce n'était pas nécessaire de mettre un nom à cette page, ni de dire à quelle date vous l'aviez créée, ou quelle taille elle faisait, que c'était vous qui l'aviez écrite, etc. Alors que si vous ouvrez votre traitement de texte, que vous tapez un texte, que vous voulez l'enregistrer, à partir de là, on vous demande de produire des textes sur ce texte, qui seront les clés qui permettront ensuite de le retrouver : le nom du fichier, etc. De là vient l'idée que ce texte est appareillé d'un autre texte, qui est ce texte sur le texte, et que cela constitue l'une des modalités importantes de votre possibilité après de le lire. Cette modalité est analysée du point de vue d'un pouvoir économique et d'un pouvoir politique, c'est-à-dire: qui va ensuite vous donner accès à quoi. Pour ce qui concerne les moteurs de recherche : qu'est-ce qui va primer dans l'affichage des résultats, sachant qu'une partie des résultats est liée à ces métadonnées, ces textes qui n'apparaissent jamais à l'écran, mais qui sont ceux à partir desquels vont être hiérarchisés les résultats ou qualifiés des textes justement, etc.

En conclusion de l'ouvrage collectif Lire, écrire, récrire, le textiel est défini comme "un état émergeant de la "forme-texte" entendue comme ensemble matériel et organisé de signes à l'écran. » (Davallon et al. [2003b] 2013, § 39). Quinze ans plus tard, en proposeriez-vous une autre définition?

J'avoue que je ne sais pas, mais je ne pense pas (le concept de textiel a justement l'intérêt d'être assez générique); probablement pas pour ce qu'il désignait pour nous à l'époque car, comme je l'ai dit précédemment, il a une forme de généricité qui permet de penser les objets numériques dans ce qu'ils ont de radicalement différent des inscriptions dans d'autres matérialités. Le textiel me semble bien traduire le fait que ces textes soient "agissables » et que l'activité interprétative et agissante du lecteur va au-delà de sa contribution à la construction du sens par la lecture, le feuilletage, l'annotation, l'action sur le dispositif de lecture. L'illettrisme numérique correspondrait pour moi à une difficulté à s'emparer de cette dimension des écrits d'écran ; c'est une question sur laquelle Dominique Cotte a beaucoup travaillé (Cotte 2002, 2004). Pour comprendre le sens des textes, il faut d'abord être capable de les mettre dans des contextes éditoriaux et des contextes sociaux qui leur donnent sens, et cela fait partie de la difficulté à s'approprier des textiels, c'est-à-dire ne pas comprendre ce qu'on peut faire avec. Si l'on se réfère à l'article de Dominique Cotte, quand on dit «Où je clique là ? ", c'est qu'on ne sait pas ce que l'on est en train de faire, on ne comprend pas la proposition textuelle qui vous est faite. 


\section{BIBLIOGRAPHIE}

Merci de votre contribution!

Adam, Jean-Michel (2011). La linguistique textuelle : introduction à l'analyse textuelle des discours. Paris : Armand Colin.

Adam, Jean-Michel dir. (2015). Faire texte : frontières textuelles et opérations de textualisation. Besançon : Presses universitaires de Franche-Comté.

Bonaccorsi, Julia, et Yves Jeanneret (2019). « Genette et les SIC, une filiation paradoxale?» Communication \& langages 202 (4), 49-66.

Bush, Vannevar (1945). « As We May Think ». Life Magazine, 9 octobre 1945.

Cotte, Dominique (2002). « L'approche néophyte de la page web, ou "Mais où je clique là ?" ", Les Cahiers du numérique 3 (3), 17-32.

Cotte, Dominique (2004). « Leurres, ruses et désorientation dans les écrits de réseau. La métis à l'écran ». Communication et langages 139 (1), 63-74. https://doi.org/10.3406/colan.2004.3254

Davallon, Jean, éd. (2012). L'économie des écritures sur le web / Volume 1 : traces d'usage dans un corpus de sites de tourisme. Paris : Hermès-Lavoisier.

Davallon, Jean, Marie Després-Lonnet, Yves Jeanneret, Joëlle Le Marec, et Emmanuël Souchier. (2013 [2003a]). Lire, écrire, récrire : Objets, signes et pratiques des médias informatisés. Études et recherche. Paris : Éditions de la Bibliothèque publique d'information. http:// books.openedition.org/bibpompidou/394.

Davallon, Jean, Marie Després-Lonnet, Yves Jeanneret, Joëlle Le Marec, et Emmanuël Souchier. (2013 [2003a]). «Conclusion ». In Lire, écrire, récrire: Objets, signes et pratiques des médias informatisés, 303-22. Études et recherche. Paris : Éditions de la Bibliothèque publique d'information. http://books.openedition.org/bibpompidou/424

Davallon, Jean, Nathalie Noël-Cadet, et Danièle Brochu. (2013 [2003a]). « Chapitre I. L'usage dans le texte : les "traces d'usage" du site Gallica ». In Lire, écrire, récrire: Objets, signes et pratiques des médias informatisés, par Marie Després-Lonnet, Yves Jeanneret, Joëlle Le Marec, et Emmanuël Souchier, 47-90. Études et recherche. Paris : Éditions de la Bibliothèque publique d'information. http://books.openedition.org/bibpompidou/409

Després-Lonnet, Marie, et Dominique Cotte (2007). « La sémiotisation d'une pratique professionnelle. L'activité de montage numérique dans l'audiovisuel ». In L'écriture des médias informatisés : espaces de pratiques, édité par Cécile Tardy et Yves Jeanneret. Paris : Hermès science. Jeanneret, Yves (2014). Critique de la trivialité: Les médiations de la communication, enjeu de pouvoir. Paris : Editions Non Standard.

Souchier, Emmanuël, et Yves Jeanneret (1999). « Pour une poétique de "l'écrit d'écran" ». Xoana, $n^{\circ} 6 / 7: 97-107$.

Tardy, Cécile, et Yves Jeanneret, éd. (2007). L'écriture des médias informatisés : espaces de pratiques. Paris : Hermès science. 


\section{NOTES}

1. Després-Lonnet Marie, Temps et lieux de la documentation. Transformation des contextes interprétatifs à l'ère d'internet, Mémoire d'habilitation à diriger des recherches, Université de Lille, $\underline{2014}$

2. Un logiciel de PAO (Publication Assistée par Ordinateur) sert à la mise en page et la structuration d'ouvrages imprimés (brochures, catalogues, etc.)

3. Cette définition renvoie à celle de Jeanneret et Souchier: "Nous nommons architextes (de archè, origine et commandement), les outils qui permettent l'existence de l'écrit d'écran et qui, non contents de représenter la structure du texte, en commandent l'exécution et la réalisation. Autrement dit, le texte naît de l'architexte qui en balise l'écriture. » (Souchier et Jeanneret 1999, 103). La notion d'architexte, popularisée par les travaux de Genette, prend en SIC une dimension qui dépasse l'étude des oeuvres littéraires où la distinction entre texte et paratexte est apparue pertinente - distinction généralement récusée par les SIC: ainsi, Jeanneret considère que la notion de paratexte ne proposerait qu'une conception restreinte de l'énonciation éditoriale (Jeanneret 2014, 123 et 137). Sur le transfert des travaux de Genette dans les SIC, voir (Jeanneret et Bonaccorsi 2019).

4. Voir Bush 1945.

\section{AUTEURS}

\section{INGRID MAYEUR}

Université de Liège

MARIE DESPRÈS-LONNET

Université Lyon 2, ELICO 